\title{
A NEW DESINGULARIZATION FOR VORTEX METHODS
}

\author{
THOMAS Y. HOU
}

\begin{abstract}
A new desingularization is introduced for the vortex method. The idea is to subtract off the most singular part in the discrete approximation to the velocity integral and replace it by the velocity of a vortex patch of constant vorticity, which can be evaluated explicitly. Stability and convergence of the method are obtained in the maximum norm. Preliminary numerical results are presented.
\end{abstract}

\section{INTRODUCTION}

In this paper, we introduce a new desingularization for the vortex method. The idea is to subtract off the most singular part in the discrete approximation to the velocity integral and replace this singular summation by the velocity of a vortex patch of constant vorticity, which can be evaluated explicitly (see the Appendix). As a consequence, the desingularized vortex method is asymptotically one order less singular than the original vortex method if the vorticity field is Lipschitz continuous. This desingularization technique applies to both the point vortex method and the vortex blob method. Preliminary numerical experiments seem to indicate that the desingularized vortex method provides more accurate approximations for large-time calculations than the original vortex method.

Because of our desingularization, we can prove stability of the method in the maximum norm. This allows us to analyze convergence of the method in the case when a local regridding procedure is introduced for the method [11]. Moreover, our analysis only uses the fact that the Biot-Savart kernel is $L_{\text {loc }}^{1}$. This implies that the method is also convergent for the analogue of the incompressible Euler equations (1)-(2) (see below) with a kernel $K$ which is more singular than the Biot-Savart kernel.

Consider the incompressible 2-D Euler equations in the vorticity-stream function formulation:

$$
\omega_{t}+(u \cdot \nabla) \omega=0, \quad \omega(x, 0)=\omega_{0}(x) .
$$

Here the velocity $u$ is related to the vorticity $\omega$ by the Biot-Savart law

$$
u(x, t)=\int_{R^{2}} K(x-y) \omega(y, t) d y,
$$

Received December 18, 1989; revised June 21, 1990.

1991 Mathematics Subject Classification. Primary 65M25; Secondary 76C05.

Key words and phrases. Vortex method, desingularization, large time accuracy.

Research supported in part by the Air Force Office of Scientific Research under URI grant AFOSR 86-0352. 
and $K$ is the Biot-Savart kernel

$$
K(x)=\frac{1}{2 \pi|x|^{2}}\left(-x_{2}, x_{1}\right)
$$

The flow trajectory $X(t, \alpha)$, defined as below, plays an important role in the vortex method. It describes the position of a fluid particle at time $t$ which starts at the position $\alpha$ initially, and satisfies the following ordinary differential equations

$$
\frac{d X}{d t}(t, \alpha)=u(X(t, \alpha), t), \quad X(0, \alpha)=\alpha .
$$

It follows from (1), (2), and (4) that

$$
\omega(X(t, \alpha), t)=\omega(\alpha, 0)=\omega_{0}(\alpha)
$$

and

$$
u(x, t)=\int_{R^{2}} K(x-X(t, \alpha)) \omega_{0}(\alpha) d \alpha .
$$

The simplest approximation of (4) and (6) consists of replacing the velocity integral by the trapezoidal rule approximation and solving the resulting ordinary differential equations:

$$
\begin{gathered}
\frac{d X_{i}^{h}}{d t}(t)=u^{h}\left(X_{i}^{h}(t), t\right), \quad X_{i}^{h}(0)=\alpha_{i}, \\
u^{h}\left(X_{i}^{h}(t), t\right)=\sum_{\substack{j \in Z^{2} \\
j \neq i}} K\left(X_{i}^{h}(t)-X_{j}^{h}(t)\right) \omega_{j} h^{2},
\end{gathered}
$$

where $\alpha_{i}=i \cdot h=\left(i_{1} h, i_{2} h\right)$ and $\omega_{j}=\omega_{0}\left(\alpha_{j}\right)$. Numerical discretization of equations (7) and (8) is known as the point vortex method [16].

However, the point vortex method has the following difficulty: if two neighboring particles approach each other, the discrete velocity approximation may become unbounded. In order to alleviate this difficulty, Chorin [5] introduced the idea of replacing (7)-(8) by the system of equations

$$
\begin{gathered}
\frac{d X_{i}^{\delta}(t)}{d t}=u^{\delta}\left(X_{j}^{\delta}(t), t\right), \quad X_{i}^{\delta}(0)=\alpha_{i}, \\
u^{\delta}\left(X_{i}^{\delta}(t), t\right)=\sum_{j \in Z^{2}} K_{\delta}\left(X_{i}^{\delta}(t)-X_{j}^{\delta}(t)\right) \omega_{j} h^{2},
\end{gathered}
$$

where $K_{\delta}$ is a regularized kernel obtained by convolving $K$ with a mollifier $f_{\delta}$,

$$
K_{\delta}=K * f_{\delta}, \quad f_{\delta}(x)=\frac{1}{\delta^{2}} f\left(\frac{x}{\delta}\right) .
$$

This gives a computationally more stable method than the point vortex method if the smoothing blob size, $\delta$, is much larger than the grid size, $h$. Convergence of the vortex blob method has been established first by Hald [9], and subsequently by Anderson and Greengard [1], Beale and Majda [3], Cottet [6], and Raviart [15] among others, under the assumption $\delta \gg h$. For a more complete bibliography, we refer to the review papers of Leonard [12] and Majda [13]. On the other hand, recently Goodman, Hou, and Lowengrub have shown 
that the point vortex method is also stable and convergent with second-order accuracy for the Euler equations with smooth solutions $[8,10]$.

However, for large-time calculations, the point vortex method and the vortex blob method with small blob size $\delta \sim h$ seem to produce relatively large errors. The purpose of this paper is to introduce a new desingularization for the vortex method so that the accuracy of the method can be maintained for relatively longer times.

\section{Desingularization FOR THE 2-D POINT VORTEX METHOD}

The new desingularization method is motivated by previous joint work with T. Chacon on a Lagrangian finite element method [4]. In the present paper, we approximate the vorticity by a piecewise linear polynomial and allow the mesh points of the triangulation to move along the streamlines of the flow. Therefore, the vorticity is calculated accurately at the mesh points, and the method is nondissipative. The main observation, which makes this formulation possible, is that the integral of a product of the Biot-Savart kernel and a polynomial over any triangle can be evaluated explicitly. More precisely, suppose $\mathrm{T}_{h}=\left\{\tau_{i}\right\}_{i=1}^{N}$ is a triangulation of the support of the vorticity, with mesh points given by the particle trajectories $\left\{X_{i}^{h}\right\}$. Let $\omega^{h}(x, t)$ be the piecewise linear approximation to $\omega(x, t)$ over $\mathrm{T}_{h}$ which satisfies the following properties:

$$
\begin{array}{ll}
\omega^{h}\left(X_{i}^{h}, t\right)=\omega_{0}\left(\alpha_{i}\right), & \omega^{h}(x, t) \text { is continuous in } x \\
& \text { and }\left.\omega^{h}\right|_{\tau_{i}} \text { is affine, } 1 \leq i \leq N .
\end{array}
$$

Then a natural approximation of $u(x, t)$ is given by

$$
\begin{aligned}
u^{h}(x, t) & =\int_{R^{2}} K(x-y) \omega^{h}(y, t) d y \\
& =\left.\sum_{\tau_{i} \in \mathrm{T}_{h}} \int_{\tau_{i}} K(x-y) \omega^{h}\right|_{\tau_{i}}(y, t) d y .
\end{aligned}
$$

Since $\left.\omega^{h}\right|_{\tau_{i}}$ is affine, the integrals which appear in (11) can be calculated analytically. Thus, we obtain a system of equations for $X_{i}^{h}(t)$ as follows:

$$
\frac{d X_{i}^{h}(t)}{d t}=\left.\sum_{\tau_{i} \in \mathrm{T}_{h}} \int_{\tau_{i}} K\left(X_{i}^{h}-y\right) \omega^{h}\right|_{\tau_{i}}(y, t) d y .
$$

A key observation is that we can reformulate the above discretization by splitting the velocity integral into two parts:

$$
\begin{aligned}
\frac{d X_{i}^{h}(t)}{d t}= & \int_{\Omega_{h}(t)} K\left(X_{i}^{h}-y\right)\left(\omega^{h}(y, t)-\omega^{h}\left(X_{i}^{h}, t\right)\right) d y \\
& +\omega^{h}\left(X_{i}^{h}, t\right) \int_{\Omega_{h}(t)} K\left(X_{i}^{h}-y\right) d y,
\end{aligned}
$$

where $\Omega_{h}(t)$ is the support of $\omega_{h}(y, t)$ at time $t$. Obviously, the integrand $K\left(X_{i}^{h}-y\right)\left(\omega^{h}(y, t)-\omega^{h}\left(X_{i}^{h}, t\right)\right)$ is bounded if $\omega^{h}$ is Lipschitz continuous. The second integral on the right of (13) represents the velocity of a vortex patch of constant vorticity. It can be evaluated explicitly by expressing the computational support $\Omega_{h}(t)$ as a union of triangles and using our explicit formula (see the Appendix). 
Applying this idea to the vortex method, we obtain the following desingularized vortex method:

$$
\begin{aligned}
\frac{d X_{i}^{h}(t)}{d t} & =\sum_{\substack{\alpha_{j} \in \Omega_{h}(0) \\
j \neq i}} K\left(X_{i}^{h}-X_{j}^{h}\right)\left(\omega_{j}-\omega_{i}\right) h^{2}+\omega_{i} \int_{\Omega_{h}(t)} K\left(X_{i}^{h}-y\right) d y \\
& \equiv u^{h}\left(X_{i}^{h}, t\right),
\end{aligned}
$$

where $\Omega_{h}(t)$ is the computational support of $\omega(y, t)$ at time $t$. Again, the integral on the right of (14) can be evaluated explicitly. This is our desingularized point vortex method. As we can see, for Lipschitz continuous vorticity, $K\left(X_{i}^{h}-X_{j}^{h}\right)\left(\omega_{j}-\omega_{i}\right)$ is one order less singular than the original summand $K\left(X_{i}^{h}-X_{j}^{h}\right) \omega_{j}$.

We note that the discrete sum on the right of (14) is an approximation to the continuous integral

$$
\int_{\Omega_{h}(0)} K\left(X_{i}^{h}(t)-X(t, \alpha)\right)\left(\omega_{0}(\alpha)-\omega_{0}\left(\alpha_{i}\right)\right) d \alpha .
$$

One feature, which is different from the original vortex method, is that the integrand no longer has compact support in $\alpha$. Therefore, we need to choose the initial computational support $\Omega_{h}(0)$ and the initial grid points $\alpha_{j}$ in such a way that the discrete sum approximates the integral over $\Omega_{h}(0)$ as accurately as possible. For example, the composite midpoint rule would lead to second-order accurate approximation if the initial support is taken to be a square. If the size of the square is $R$, then $\alpha_{j}=\left(j_{1}+\frac{1}{2}, j_{2}+\frac{1}{2}\right) h, h=R / M$ for some integer $M>0$.

Convergence of the method can be obtained by a local argument. Actually, the desingularization allows us to prove stability of the method in the maximum norm. To be specific, we present a proof in the case when we approximate the support of the vorticity by a polygonal domain and use a midpoint rule approximation for the integral (15).

Theorem 1. Suppose that $\omega_{0} \in C_{0}^{2}\left(R^{2}\right)$, and the support of $\omega(x, t)$ is approximated by a polygonal domain. Further, assume that a midpoint rule discretization is used in approximating the integral (15). Then the solutions of the desingularized vortex method (14) satisfy

$$
\begin{gathered}
\left\|X^{h}(t)-X(t)\right\|_{l_{\infty}} \leq C h^{2}|\log (h)|, \\
\left\|u^{h}\left(X^{h}(t), t\right)-u(X(t), t)\right\|_{l_{\infty}} \leq C h^{2}|\log (h)| \text { for } 0 \leq t \leq T .
\end{gathered}
$$

Remark 1. Our desingularized method does not require that $\Omega_{h}(0)$ be the exact support of the initial vorticity. In computations, we might take a larger initial computational support $\Omega_{h}(0)$ than the actual support of $\omega_{0}(x)$.

Proof of Theorem 1. As usual, we divide the proof into two parts: consistency 
and stability. We first estimate the consistency error $\sigma$ :

$$
\begin{array}{r}
\sigma=u\left(X_{i}(t), t\right)-\left(\sum_{\substack{\alpha_{j} \in \Omega_{h}(0) \\
j \neq i}} K\left(X_{i}(t)-X_{j}(t)\right)\left(\omega_{j}-\omega_{i}\right) h^{2}\right. \\
\left.+\omega_{i} \int_{\Omega_{h}^{c}(t)} K\left(X_{i}(t)-y\right) d y\right),
\end{array}
$$

where $\Omega_{h}^{c}(t)$ is the approximation of the exact support $\Omega(t)$ using the exact particle trajectories $X_{j}(t)$. It is natural to split $\sigma$ into two terms

$$
\sigma=\sigma_{1}+\sigma_{2}
$$

where

$$
\begin{aligned}
\sigma_{1}= & \int_{\Omega_{h}(0)} K\left(X_{i}(t)-X(t, \alpha)\right)\left(\omega_{0}(\alpha)-\omega_{0}\left(\alpha_{i}\right)\right) d \alpha \\
& -\sum_{\substack{\alpha_{j} \in \Omega_{h}(0) \\
j \neq i}} K\left(X_{i}(t)-X_{j}(t)\right)\left(\omega_{j}-\omega_{i}\right) h^{2}
\end{aligned}
$$

and

$$
\sigma_{2}=\omega_{i} \int_{\Omega(t)} K\left(X_{i}(t)-y\right) d y-\omega_{i} \int_{\Omega_{h}^{c}(t)} K\left(X_{i}(t)-y\right) d y .
$$

Denote by $B_{R}$ the square centered at the origin with size $R$. Without loss of generality, we assume that the support of $\omega_{0}$ is contained in $B_{R}$. To simplify our analysis, we take a larger computational support to start with, i.e., $\Omega_{h}(0)=$ $B_{R+2}$.

For the $\sigma_{1}$-term, we apply the standard error estimate for the midpoint rule. Let $B_{j}$ be the square centered at $\alpha_{j}$ with size $h$. We have

$$
\left|\sigma_{1}\right| \leq h^{2} \sum_{j \neq i} \max _{\alpha \in B_{j}}\left|\partial_{\alpha}^{2} F(\alpha)\right| h^{2}+\int_{B_{i}} F(\alpha) d \alpha,
$$

where

$$
F(\alpha)=K\left(X\left(t, \alpha_{i}\right)-X(t, \alpha)\right)\left(\omega_{0}(\alpha)-\omega_{0}\left(\alpha_{i}\right)\right) .
$$

Using $\left|X\left(t, \alpha_{i}\right)-X(t, \alpha)\right| \geq C\left|\alpha_{i}-\alpha\right|$, we can show that $F(\alpha)$ is bounded and

$$
\left|\sigma_{1}\right| \leq h^{2} \int_{h \leq\left|\alpha-\alpha_{i}\right| \leq R} \frac{C}{\left|\alpha-\alpha_{i}\right|^{2}} d \alpha+C h^{2} \leq C h^{2}|\log (h)| .
$$

Here, $C$ is a generic constant depending on $\omega_{0}$ and $T$ only. We will use this notation throughout the proof.

For the $\sigma_{2}$-term, there are two cases.

Case (i): $\alpha_{i} \notin B_{R}$. Then $\omega_{i}=0$, since $\omega_{0}$ has support contained in $B_{R}$. Therefore, $\sigma_{2}=0$.

Case (ii): $\alpha_{i} \in B_{R}$. Let $\Omega_{R+1}=\left\{y: y=X(t, \alpha), \alpha \in B_{R+1}\right\}$. Note that $\Omega(t)=\Omega_{R+2}$ by our assumption. Since the approximation of $\Omega(t)$ by a 
polygonal domain $\Omega_{h}(t)$ is second-order accurate, we conclude that $\Omega_{R+1}$ is contained in both $\Omega(t)$ and $\Omega_{h}^{c}(t)$. Thus, we get

$$
\sigma_{2}=\omega_{i}\left(\int_{\Omega(t) \backslash \Omega_{R+1}} K\left(X_{i}(t)-y\right) d y-\int_{\Omega_{h}^{c}(t) \backslash \Omega_{R+1}} K\left(X_{i}(t)-y\right) d y\right) .
$$

But if $y$ lies outside the region $\Omega_{R+1}$, i.e., $y=X(t, \alpha)$ with $|\alpha|>R+1$, we have

$$
\left|X\left(t, \alpha_{i}\right)-X(t, \alpha)\right| \geq C\left|\alpha_{i}-\alpha\right| \geq C,
$$

where we have used $\left|\alpha_{i}\right| \leq R$ and the fact that the inverse of $X$ is smooth by incompressibility. Consequently,

$$
\left|\sigma_{2}\right| \leq C \mid \text { area of } \Omega(t) \backslash \Omega_{R+1}-\text { area of } \Omega_{h}^{c}(t) \backslash \Omega_{R+1} \mid \leq C h^{2} .
$$

Combining (22) and (23), we obtain the following consistency estimate:

$$
|\sigma| \leq C h^{2}|\log (h)| .
$$

Now we turn to proving stability of the method. As in the consistency estimates, we split the stability error, denoted by $\rho$, into two parts

$$
\rho=\rho_{1}+\rho_{2},
$$

where

$$
\begin{aligned}
\rho_{1}= & \sum_{\substack{\alpha_{j} \in \Omega_{h}(0) \\
j \neq i}} K\left(X_{i}(t)-X_{j}(t)\right)\left(\omega_{j}-\omega_{i}\right) h^{2} \\
& -\sum_{\substack{\alpha \in \in \Omega_{h}(0) \\
j \neq i}} K\left(X_{i}^{h}(t)-X_{j}^{h}(t)\right)\left(\omega_{j}-\omega_{i}\right) h^{2}
\end{aligned}
$$

and

$$
\rho_{2}=\omega_{i} \int_{\Omega_{h}^{c}(t)} K\left(X_{i}(t)-y\right) d y-\omega_{i} \int_{\Omega_{h}(t)} K\left(X_{i}^{h}(t)-y\right) d y .
$$

Let us denote $e_{j}(t)=X_{j}(t)-X_{j}^{h}(t)$. Define

$$
T^{*}=\inf \left\{t: 0 \leq t \leq T,\|e(t)\|_{l_{\infty}} \leq h^{3 / 2}\right\} .
$$

Using the Mean Value Theorem, we get

$$
\rho_{1}=\sum_{\substack{\alpha_{j} \in \Omega_{h}(0) \\ j \neq i}} \nabla K\left(X_{i}(t)-X_{j}(t)+\xi_{i j}\right)\left(e_{i}(t)-e_{j}(t)\right)\left(\omega_{j}-\omega_{i}\right) h^{2},
$$

where $\left|\xi_{i j}\right| \leq 2\|e(t)\|_{l_{\infty}} \leq 2 h^{3 / 2}$ for $t \leq T^{*}$. The application of the mean value theorem is well justified because for $t<T^{*}$ and $i \neq j$,

$$
\begin{aligned}
\left|X_{i}(t)-X_{j}(t)+\xi_{i j}\right| & \geq\left|X\left(t, \alpha_{i}\right)-X\left(t, \alpha_{j}\right)\right|-2 h^{3 / 2} \\
& \geq C\left|\alpha_{i}-\alpha_{j}\right|-2 h^{3 / 2} \geq \frac{C}{2}\left|\alpha_{i}-\alpha_{j}\right| .
\end{aligned}
$$

This also implies that

$$
\left|\rho_{1}\right| \leq 2\|e(t)\|_{l_{\infty}} \sum_{\substack{\alpha_{j} \in \Omega_{h}(0) \\ j \neq i}} \frac{C h^{2}}{\left|\alpha_{i}-\alpha_{j}\right|} \leq C\|e(t)\|_{l_{\infty}} .
$$


To estimate $\rho_{2}$, we again divide the argument into two cases.

Case (i): $\alpha_{i} \notin B_{R}$. Then $\omega_{i}=0$ and $\rho_{2}=0$.

Case (ii): $\alpha_{i} \in B_{R}$. We write

$$
\begin{aligned}
\rho_{2}= & \left(\omega_{i} \int_{\Omega_{h}^{c}(t)} K\left(X_{i}(t)-y\right) d y-\omega_{i} \int_{\Omega_{h}^{c}(t)} K\left(X_{i}^{h}(t)-y\right) d y\right) \\
& +\left(\omega_{i} \int_{\Omega_{h}^{c}(t)} K\left(X_{i}^{h}(t)-y\right) d y-\omega_{i} \int_{\Omega_{h}(t)} K\left(X_{i}^{h}(t)-y\right) d y\right) \\
& \equiv \rho_{2}^{(1)}+\rho_{2}^{(2)} .
\end{aligned}
$$

Rewrite $\rho_{2}^{(1)}$ in the form

$$
\rho_{2}^{(1)}=\omega_{i} e_{i}(t) \int_{0}^{1} \int_{\Omega_{h}^{c}(t)} \nabla K\left(X_{i}(t)-y+\theta e_{i}(t)\right) d y d \theta .
$$

Since $\alpha_{i} \in B_{R}$ and $\Omega_{h}^{c}$ is an accurate approximation to $\Omega(t)=\{y: y=$ $\left.X(t, \alpha), \alpha \in B_{R+2}\right\}$, we conclude that there exists a constant $r_{0}$ such that $B_{i, r_{0}}=\left\{y:\left|y-X_{i}(t)-\theta e_{i}(t)\right| \leq r_{0}\right\}$ is contained in $\Omega_{h}^{c}(t)$ for $t<T^{*}$. But the integration of $\nabla K$ over a symmetric domain, $B_{i, r_{0}}$, is equal to zero, and outside $B_{i, r_{0}}, \nabla K$ is bounded. This implies that

$$
\left|\rho_{2}^{(1)}\right| \leq C\|e\|_{l_{\infty}} \text { for } t<T^{*} .
$$

For the $\rho_{2}^{(2)}$-term, we can argue exactly as we did for $\sigma_{2}$ (Case (ii)) in the consistency estimate. We get

$$
\begin{aligned}
\left|\rho_{2}^{(2)}\right| & \leq C \mid \text { area of } \Omega_{h}^{c}(t) \backslash \Omega_{R+1}-\text { area of } \Omega_{h}(t) \backslash \Omega_{R+1} \mid \\
& \leq C\|e(t)\|_{l_{\infty}} \text { for } t<T^{*} .
\end{aligned}
$$

This completes our stability estimate

$$
|\rho| \leq C\|e(t)\|_{l_{\infty}} \text { for } 0 \leq t<T^{*} .
$$

Combining (24) and (31), we arrive at

$$
\frac{d}{d t}\|e(t)\|_{l_{\infty}} \leq C\left(\|e(t)\|_{l_{\infty}}+h^{2}|\log (h)|\right)
$$

for $t<T^{*}$. The Gronwall lemma then implies

$$
\|e(t)\|_{l_{\infty}} \leq C(T) h^{2}|\log (h)| .
$$

For $h$ small enough, we have $C(T) h^{2}|\log (h)| \leq \frac{1}{2} h^{3 / 2}$. This shows that $T^{*}=$ $T$, and inequality (33) holds for all $t \leq T$. This proves (16). Now, it follows from (32) that

$$
\left\|u^{h}\left(X_{i}^{h}(t), t\right)-u\left(X_{i}(t), t\right)\right\|_{l_{\infty}}=\left\|\frac{d}{d t} e(t)\right\|_{l_{\infty}} \leq C\left(\|e(t)\|_{l_{\infty}}+h^{2}|\log (h)|\right),
$$

which implies (17) in light of (33). This completes the proof of Theorem 1.

Remark 2. As we see in the proof of Theorem 1, we have only used the fact that the kernel $K$ is $L_{\text {loc }}^{1}$. Thus, our result applies to the analogue of the 
incompressible 2-D Euler equations with a kernel $K$ which is more singular than the Biot-Savart kernel.

\section{Desingularization For the 2-D vORTEX BLOB METHOD}

The desingularization scheme presented in $\S 2$ can be extended to the vortex blob method. Consider the regularized 2-D Euler equations

$$
\omega_{t}^{\delta}+u^{\delta} \cdot \nabla \omega^{\delta}=0, \quad \omega^{\delta}(x, 0)=\omega_{0}(x),
$$

and

$$
u^{\delta}(x, t)=K_{\delta} * \omega,
$$

where $K_{\delta}=K * \phi_{\delta}$ is a regularized kernel, $\phi_{\delta}=\phi(x / \delta) / \delta^{2}$, and $\phi$ is a smooth $m$ th-order cutoff function satisfying

(i) $\int \phi(x) d x=1$;

(ii) $\int \phi(x) x^{\alpha} d x=0$ for all multi-indices $|\alpha| \leq m-1$;

(iii) $\phi(x)=\phi(|x|)$, and $\phi(x)=0$ for $|x| \geq 1$.

It is well known that for an $m$ th-order cutoff function (see, e.g., [3]),

$$
\left|\omega(x, t)-\omega^{\delta}(x, t)\right| \leq C \delta^{m}, \quad\left|u(x, t)-u^{\delta}(x, t)\right| \leq C \delta^{m} .
$$

Clearly, the generalization of the desingularized scheme (14) to the equation (34) is given by

$$
\frac{d X_{i}^{\delta}(t)}{d t}=\sum_{\alpha_{j} \in \Omega_{h}(0)} K_{\delta}\left(X_{i}^{\delta}-X_{j}^{\delta}\right)\left(\omega_{j}-\omega_{i}\right) h^{2}+\omega_{i} \int_{\Omega_{h}(t)} K_{\delta}\left(X_{i}^{\delta}-y\right) d y,
$$

where $\Omega_{h}(t)$ is the computational support of $\omega(y, t)$ at time $t$, and $\omega_{j}=$ $\omega_{0}\left(\alpha_{j}\right)$. The question is how to compute the patch velocity corresponding to $K_{\delta}$. Let us denote by $\Omega(t)$ the support of the exact vorticity $\omega(x, t)$. We claim that if we take an initial computational support $\Omega_{h}(0)$ large enough so that

$$
\operatorname{dist}\left(\partial \Omega_{h}(t), \partial \Omega(t)\right) \geq 2 \delta,
$$

then

$$
\omega_{i} \int_{\Omega_{h}(t)} K_{\delta}\left(X_{i}^{\delta}-y\right) d y=\omega_{i} \int_{\Omega_{h}(t)} K\left(X_{i}^{\delta}-y\right) d y .
$$

The patch velocity can then be evaluated analytically by our explicit formula in the Appendix.

We prove (37) as follows. If $\alpha_{i}$ lies outside the initial vorticity support, i.e., $\alpha_{i} \notin \Omega(0)$, then $\omega_{i}=0$ and therefore (37) is valid. If $\alpha_{i} \in \Omega(0)$, the assumption on $\Omega_{h}(0)$ implies that the circle centered at $X_{i}^{\delta}$ with radius $\delta$ is embedded completely inside $\Omega_{h}(t)$. Now, recall that $K_{\delta}(x)=K(x) \int_{|y| \leq|x| / \delta} \phi(y) d y$ (see, e.g., $[2,10])$. We conclude that $K_{\delta}(x)=K(x)$ if $|x| \geq \delta$, and $K_{\delta}(x)$ is an odd function in $x$. These two facts together imply (37), since the integrals of both $K$ and $K_{\delta}$ over the circle centered at $X_{i}^{\delta}$ vanish.

In view of (37), our desingularized vortex blob method becomes

$$
\begin{aligned}
\frac{d X_{i}^{\delta}(t)}{d t} & =\sum_{\alpha_{j} \in \Omega_{h}(0)} K_{\delta}\left(X_{i}^{\delta}-X_{j}^{\delta}\right)\left(\omega_{j}-\omega_{i}\right) h^{2}+\omega_{i} \int_{\Omega_{h}(t)} K\left(X_{i}^{\delta}-y\right) d y \\
& \equiv u_{\delta}\left(X_{i}^{\delta}, t\right),
\end{aligned}
$$


where $\Omega_{h}(0)$ is taken large enough so that (36) is satisfied. In practice, this can be achieved by adding particles outside the vorticity support adaptively.

Arguing as in the proof of Theorem 1, we can prove the following convergence theorem for the desingularized vortex blob method (38).

Theorem 2. Suppose that an mth-order cutoff function is used and (36) is satisfied. Under the assumption of Theorem 1, the solutions of the desingularized vortex blob method (38) satisfy

$$
\text { (40) }\left\|u^{\delta}\left(X^{\delta}(t), t\right)-u(X(t), t)\right\|_{l_{\infty}} \leq C\left(\delta^{m}+h^{2}|\log (h)|\right) \text { for } 0 \leq t \leq T \text {. }
$$

$$
\left\|X^{\delta}(t)-X(t)\right\|_{l_{\infty}} \leq C\left(\delta^{m}+h^{2}|\log (h)|\right),
$$

Remark 3. It was pointed out by Lowengrub and Shelley (see [11]) that the patch velocity term on the right of (38) can be eliminated by making the desingularization local. This amounts to mollifying the constant vortex patch by a radially symmetric cutoff function, i.e., replacing $\omega_{i}$ by $\omega_{i} \phi\left(X_{i}^{\delta}-X_{j}^{\delta}\right)$ in (38). The patch velocity vanishes because of the oddness of the integrand. Moreover, with this smooth cutoff, the desingularized vortex blob method is high-order accurate $O\left(\delta^{m}+(h / \delta)^{r} \delta^{2}\right)$ [11], where $r$ is the degree of smoothness of the flow.

\section{Generalization to the 3-D Euler equations}

In this section, we would like to generalize the desingularization idea to the 3-D Euler equations. The hope is that through desingularization the stretching term can be approximated in a more stable manner.

The 3-D incompressible Euler equations in the vorticity-stream function formulation are given by

$$
\omega_{t}+u \cdot \nabla \omega=(\omega \cdot \nabla) u
$$

and

$$
u(x, t)=\int K(x-y) \omega(y, t) d y
$$

where

$$
K(x)=-\frac{1}{4 \pi|x|^{3}}\left(\begin{array}{ccc}
0 & x_{3} & -x_{2} \\
-x_{3} & 0 & x_{1} \\
x_{2} & -x_{1} & 0
\end{array}\right) .
$$

Formal application of our desingularization idea would lead to the following grid-free vortex method:

$$
\begin{aligned}
\frac{d X_{i}^{\delta}(t)}{d t}= & \sum_{\alpha_{j} \in \Omega_{h}(0)} K_{\delta}\left(X_{i}^{\delta}(t)-X_{j}^{\delta}(t)\right)\left(\omega_{j}^{\delta}(t)-\omega_{i}^{\delta}(t)\right) h^{3} \\
& +\omega_{i}^{\delta}(t) \int_{\Omega_{h}(t)} K\left(X_{i}^{\delta}(t)-y\right) d y \\
\frac{d \omega_{i}^{\delta}(t)}{d t}= & \omega_{i}^{\delta}(t) \cdot \sum_{\alpha_{j} \in \Omega_{h}(0)} \nabla K_{\delta}\left(X_{i}^{\delta}(t)-X_{j}^{\delta}(t)\right)\left(\omega_{j}^{\delta}(t)-\omega_{i}^{\delta}(t)\right) h^{3} \\
& +\omega_{i}^{\delta}(t) \cdot \int_{\Omega_{h}(t)} \nabla K\left(X_{i}^{\delta}(t)-y\right) d y
\end{aligned}
$$


where $\Omega_{h}(t)$ is the computational support of $\omega(y, t)$ at time $t$. The point vortex method corresponds to $\delta=0$. For the vortex blob method, the restriction (36) is imposed on $\Omega_{h}(t)$. In principle, by using spherical coordinates, the integral of $K$ or $\nabla K$ over any triangle can be reduced to an integral of rational type, which can be evaluated explicitly. However, the implementation of such an algorithm is more complicated than its 2-D analogue.

Convergence of the desingularized 3-D vortex method (44)-(45) can be analyzed either by following Beale's proof for the vortex blob method [2], or by following the proof of Cottet, Goodman, and Hou [7]. In either case, the stability can be obtained more easily in our case since the integrand is less singular. In particular, the growing factor $\log (1 / h)$, which appeared in the stability estimate of [7], can be eliminated.

From a computational point of view, the distortion of the Lagrangian particle trajectories in time will eventually reduce the accuracy of the discretization, no matter how accurately one can follow the particle trajectories. Thus, local regridding seems unavoidable for a large-time calculation. In a subsequent paper [11] we will analyze the convergence property of our desingularized vortex method with local regridding. In this case, the stability in the maximum norm is crucial in making a local analysis.

\section{NUMERICAL RESULTS}

In this section, we present some preliminary numerical tests that exhibit the practical performance of our desingularized vortex method. Second-order convergence is illustrated numerically. In particular, our numerical experiments show that the numerical error for the desingularized vortex method grows more slowly than that of the original vortex method. The improvement is especially evident for the point vortex method and the vortex blob method with small blobs.

In our tests, we use the radially symmetric vorticity distribution

$$
\omega(x)= \begin{cases}\left(1-|x|^{2}\right)^{7} & \text { if }|x| \leq 1 \\ 0 & \text { if }|x|>1\end{cases}
$$

The corresponding velocity field is given by $u(x)=f(|x|)\left(-x_{2}, x_{1}\right)$, with $f(r)$ defined by

$$
f(r)= \begin{cases}\left(1-\left(1-r^{2}\right)^{8}\right) /\left(16 r^{2}\right) & \text { if } r \leq 1, \\ 1 /\left(16 r^{2}\right) & \text { if } r>1 .\end{cases}
$$

The pair $(u, \omega)$ is a steady solution of the Euler equations (1) and (2). The vorticity $\omega$ is in $C^{6}\left(R^{2}\right)$ with support contained in the unit circle.

This flow is radially symmetric and rotates about the origin. Particles with maximum speed, situated approximately on $|x|=0.4$, complete one rotation at time $t=4 \pi$. Those on $|x|=1$ complete one rotation at time $t=32 \pi$. Consequently, a large tangential stretching is produced in a relatively short time. This particular point $(u, \omega)$ has been used, among others, by M. Perlman [14] in the numerical study of the accuracy of the vortex blob method.

In this particular case, the velocity of the circular vortex patch can be written explicitly as

$$
\int_{|y| \leq 1} K(x-y) d y=\pi|x|^{2} K(x) \quad \text { for }|x| \leq 1
$$




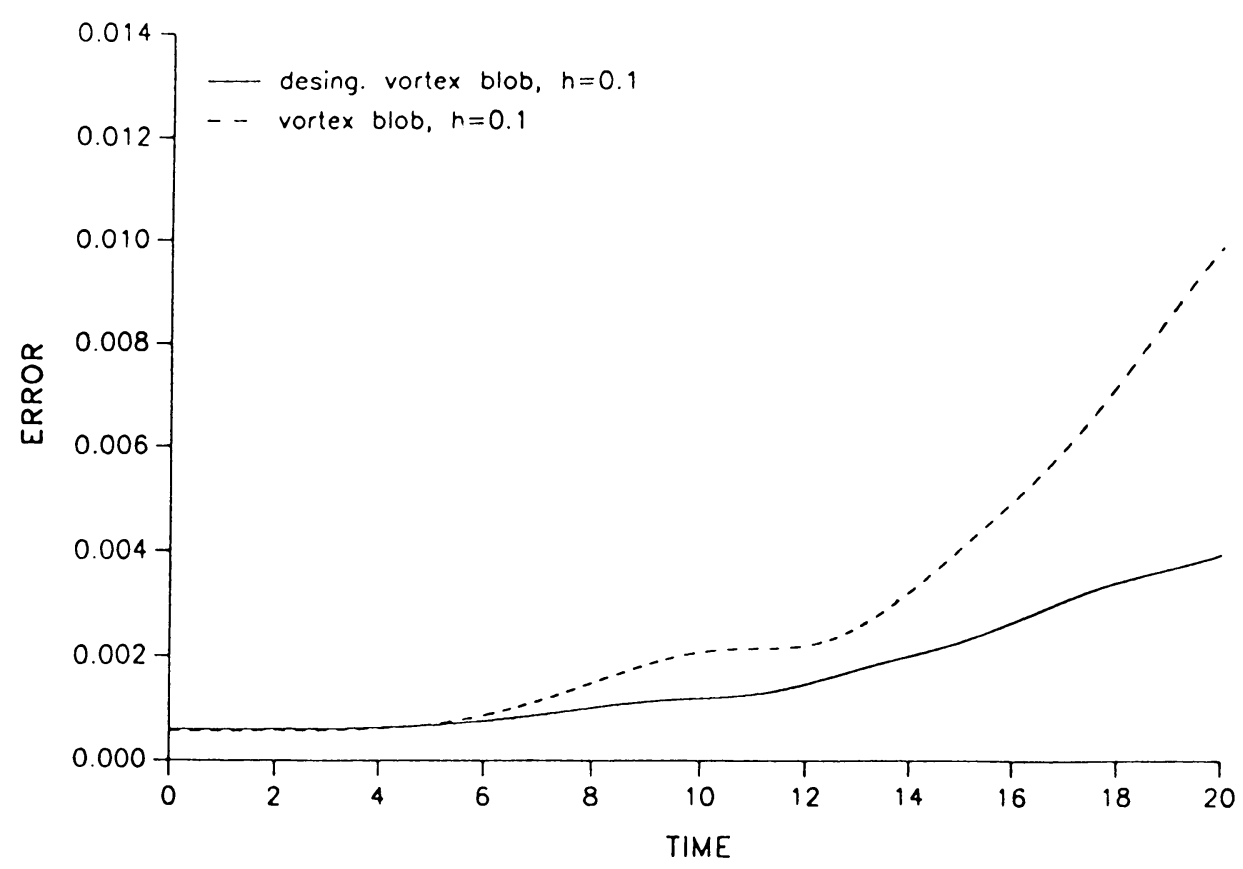

FIGURE 1

$L_{2}$ errors in velocity; desing. vortex blob vs. vortex blob, $\delta=h^{0.95}$

We plot the error in velocity both in maximum norm and discrete $l_{2}$ norm:

$$
\|e(t)\|_{l_{\infty}}=\max _{j}\left|e_{j}(t)\right|, \quad\|e(t)\|_{l_{2}}=h\left(\sum_{j}\left|e_{j}(t)\right|^{2}\right)^{1 / 2} .
$$

In Figure 1, we present the calculation using the desingularized vortex blob method with small blob size $\delta=h^{0.95}$ and compare with the calculation using the original vortex blob method. Here we use a fourth-order cutoff function. As we see, the accuracy is maintained for longer times for the desingularized vortex blob method. A similar comparison between the desingularized point vortex method and the original point vortex method is given in Figure 2. It is clear that the desingularized version provides a more accurate approximation for large times.

Figure 3 shows the errors in velocity for the desingularized point vortex method in the maximum norm for $h=0.2,0.1,0.05$, respectively. We can see that in all these calculations, the errors grow slowly in time and convergence is clearly observed as $h$ decreases.

We also compute the order of convergence of the desingularized vortex method using the following approximate formula:

$$
r(h, t)=\log _{2}(e(2 h, t) / e(h, t)),
$$

where $e(h, t)$ is the numerical error in $l_{2}$ norm at time $t$ with step size $h$. 


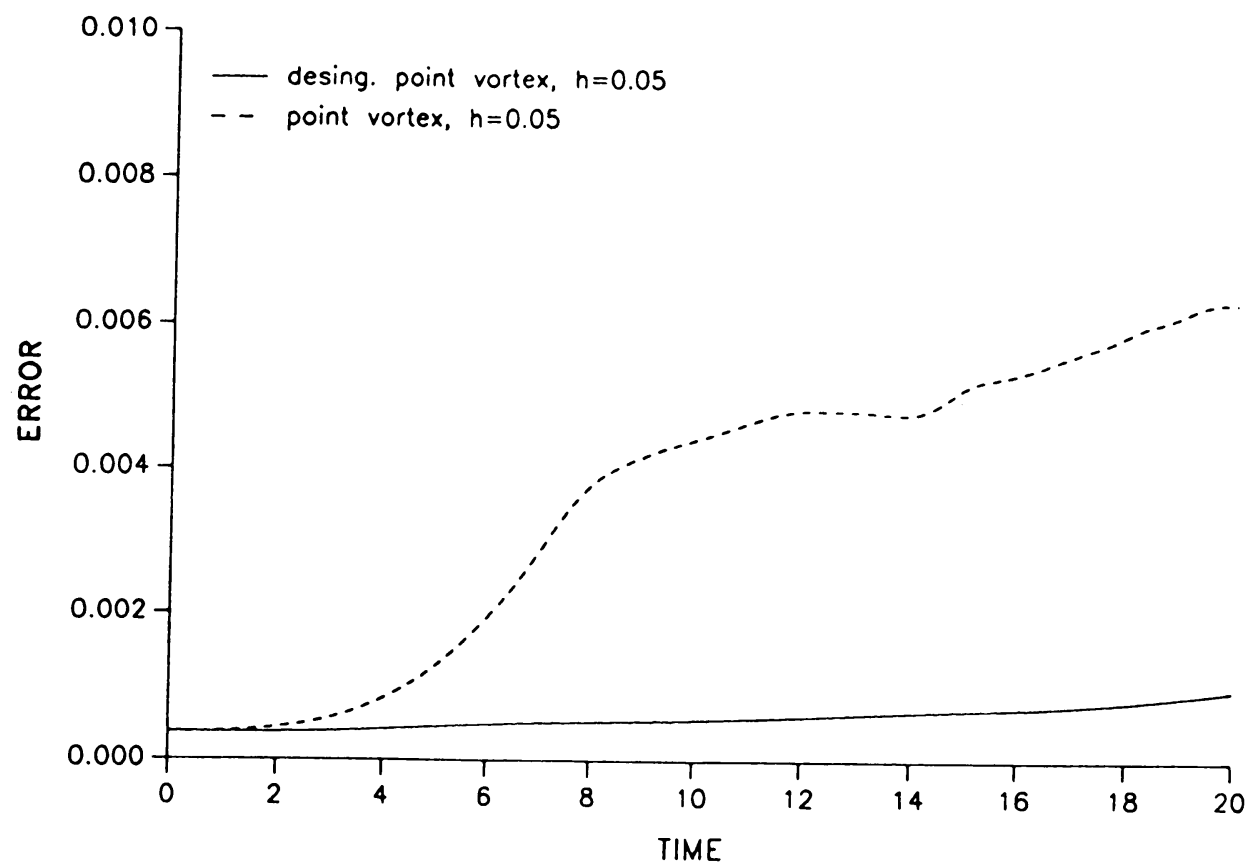

Figure 2

$L_{2}$ errors in velocity; desing. point vortex vs. point vortex

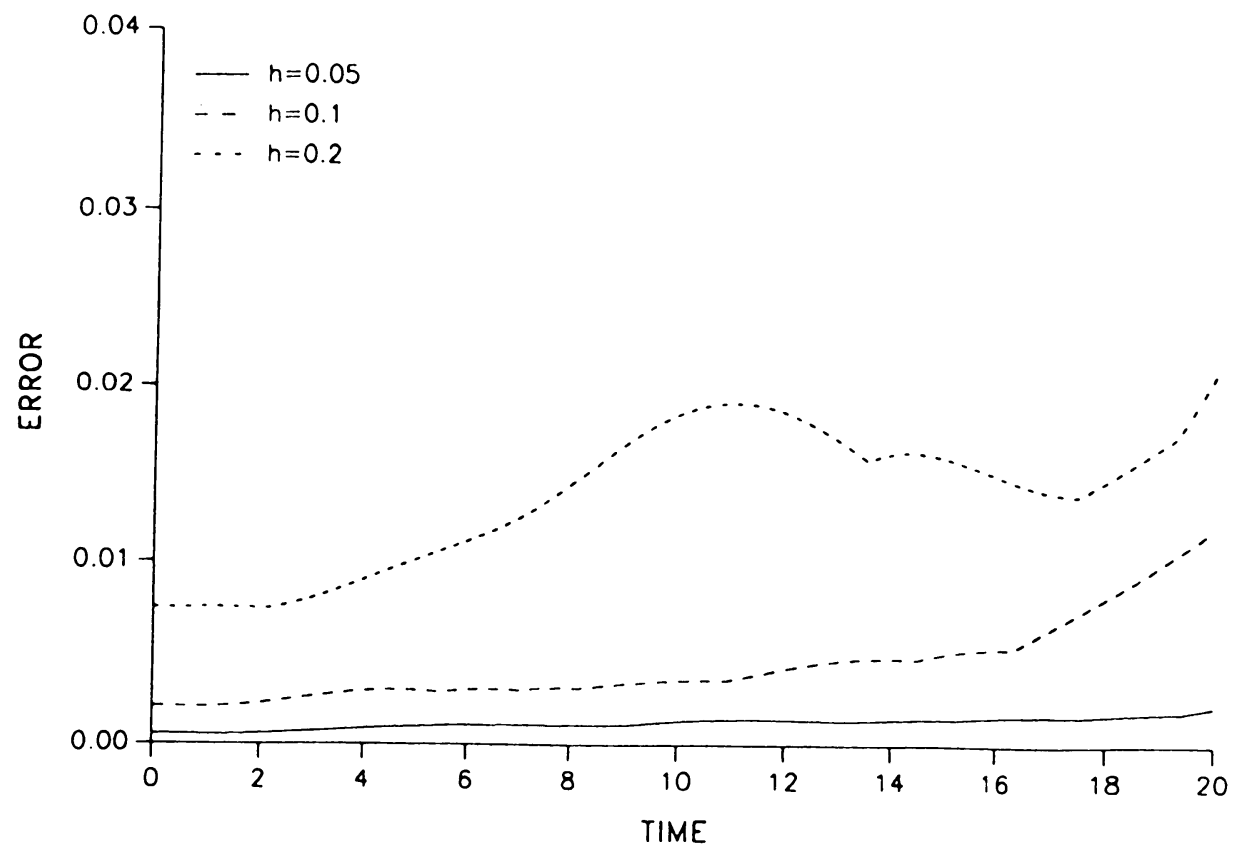

FIGURE 3

Maximum errors in velocity for desing. point vortex method

In Figure 4, we compare the order of convergence of the desingularized point vortex method with that of the corresponding residual (or truncation error) $\sigma$ (see (18)). The agreement of these two indicates that the method is stable and the stability error $\rho$ is very small. 


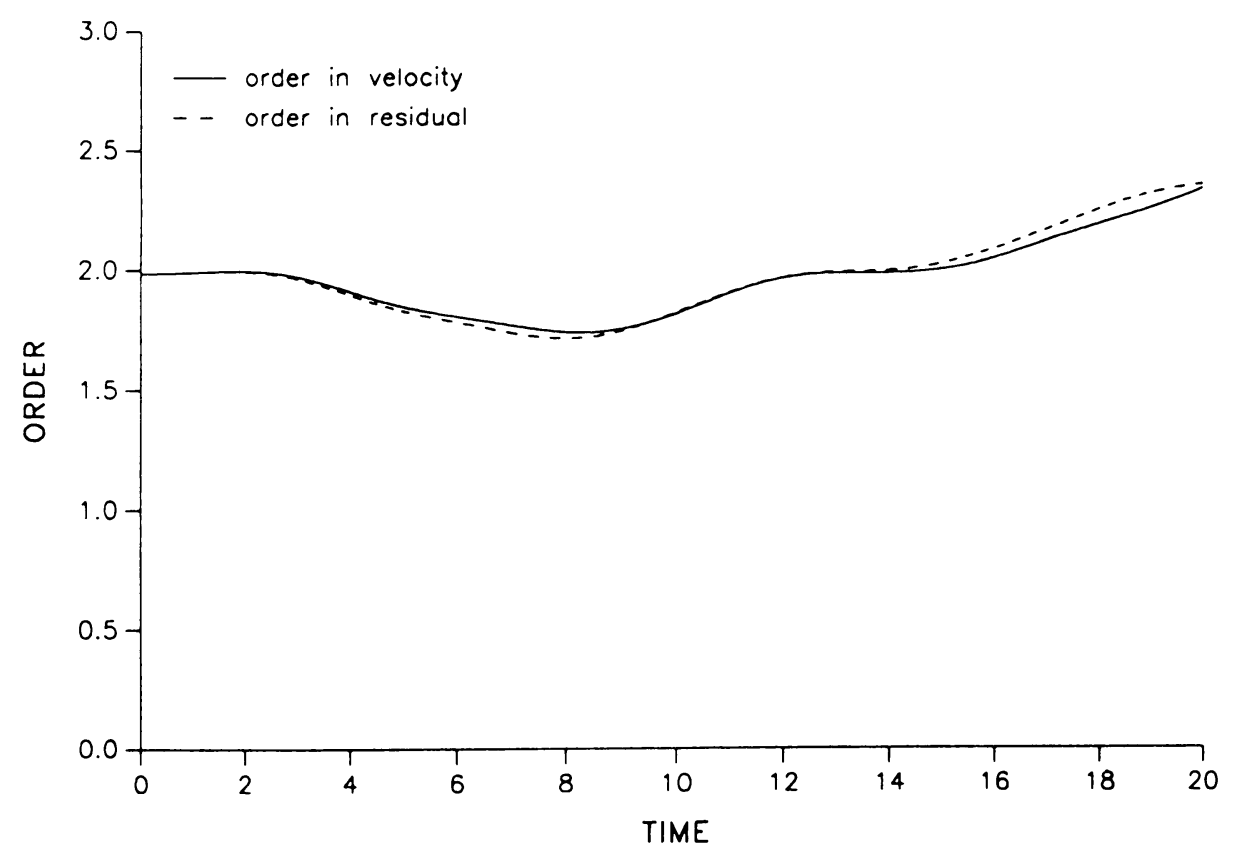

FIGURE 4

Orders of convergence for desing. point vortex method, $h=0.1,0.05$

APPENDIX

ANALYTIC CALCULATION OF VELOCITY OF A VORTEX PATCH

In this appendix we present an explicit formula to evaluate the integral of the Biot-Savart kernel $K$ over a polygonal domain $\Omega_{h}$,

$$
\int_{\Omega_{h}} K(\hat{x}-y) d y \text {. }
$$

Since one can always express the above integral as a sum of integrals over triangles with $\hat{x}$ as a vertex, it is enough to give the formula to evaluate

$$
J=\int_{\tau} K(\hat{x}-y) d y,
$$

where $\tau$ is a triangle with three vertices $\hat{x}=\left(\hat{x}_{1}, \hat{x}_{2}\right), p=\left(p_{1}, p_{2}\right)$, and $q=\left(q_{1}, q_{2}\right)$.

Let $\hat{z}$ be the orthogonal projection of $\hat{x}$ over the straight line connecting vertices $p$ and $q$, and $\beta$ be the internal angle between this straight line and the negative $y_{1}$-coordinate axis (see the figure below).

We express $J$ in polar coordinates as follows:

$$
J=\frac{1}{2 \pi} \int_{\theta_{1}}^{\theta_{2}} \int_{0}^{\rho_{\theta}}(\sin \theta,-\cos \theta) d \rho d \theta
$$

where

(A.3) $\quad \theta_{1}=\arctan \frac{q_{2}-\hat{x}_{2}}{q_{1}-\hat{x}_{1}}, \quad \theta_{2}=\arctan \frac{p_{2}-\hat{x}_{2}}{p_{1}-\hat{x}_{1}}, \quad \rho_{\theta}=\frac{|\hat{x}-\hat{z}|}{\sin (\theta+\beta)}$. 


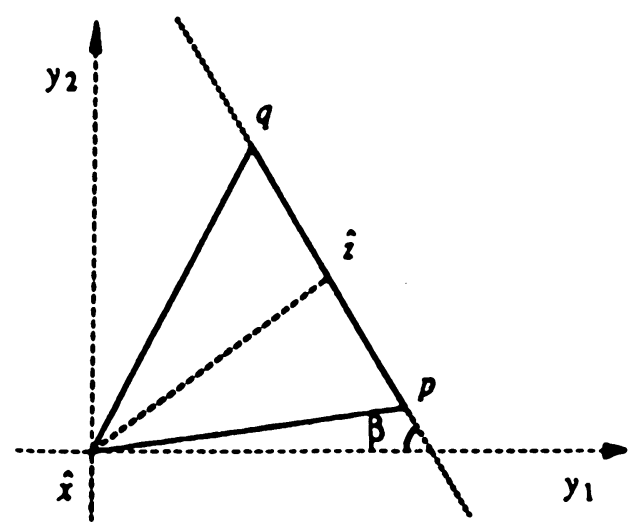

The change of variables $\zeta=\tan \theta$ gives

$$
J=\frac{1}{2 \pi} \frac{|\hat{x}-\hat{z}|}{\cos \beta} \int_{\arctan \theta_{1}}^{\arctan \theta_{2}} \frac{1}{(\zeta+\tan \beta)} \frac{1}{\left(\zeta^{2}+1\right)}(\zeta,-1) d \zeta .
$$

The integrals appearing in this expression are of rational type. Then, it is possible to find analytic expressions for $J$. A careful calculation of the integral appearing in (A.4) leads to the following formula:

$$
J=\frac{1}{2 \pi}\left[\begin{array}{l}
-d_{1} \log r+d_{2} \hat{\theta} \\
-d_{1} \hat{\theta}-d_{2} \log r
\end{array}\right],
$$

where $d_{k}=\hat{z}_{k}-\hat{x}_{k}, \hat{\theta}=\theta_{2}-\theta_{1}$, and $r=|\hat{x}-p| /|\hat{x}-q|$.

A more general formula was given in [4], which allows us to evaluate the integral of a product of the Biot-Savart kernel and a polynomial over a triangle.

\section{ACKNOWLEDGMENTS}

I would like to thank Professors Tom Beale, John Lowengrub, and Mike Shelley for some beneficial discussions. I would also like to express my gratitude to the referee for several valuable comments concerning the original manuscript.

\section{BIBLIOGRAPHY}

1. C. Anderson and C. Greengard, On vortex methods, SIAM J. Numer. Anal. 22 (1985), 413-440.

2. J. T. Beale, A convergent 3-D vortex method with grid-free stretching, Math. Comp. 46 (1986), 401-424.

3. J. T. Beale and A. Majda, Vortex methods II: High order accuracy in two and three dimensions, Math. Comp. 32 (1982), 29-52.

4. T. Chacon-Rebollo and T. Y. Hou, A Lagrangian finite element method for the 2-D Euler equations, Comm. Pure Appl. Math. 43 (1990), 735-767.

5. A. J. Chorin, Numerical study of slightly viscous flow, J. Fluid Mech. 57 (1973), 785-796.

6. G. H. Cottet, Méthodes particulaires pour l'équation d'Euler dans le plan, Thèse 3ème Cycle Univ. P. et M. Curie, 1982.

7. G. H. Cottet, J. Goodman, and T. Y. Hou, Convergence of the grid free point vortex method for the 3-D Euler equations, SIAM J. Numer. Anal. 28 (1991), 291-307.

8. J. Goodman, T. Y. Hou, and J. Lowengrub, Convergence of the point vortex method for 2-D Euler equations, Comm. Pure Appl. Math. 43 (1990), 415-430.

9. O. Hald, Convergence of vortex methods. II, SIAM J. Numer. Anal. 16 (1979), 726-755. 
10. T. Y. Hou and J. Lowengrub, Convergence of the point vortex method for the 3-D Euler equations, Comm. Pure Appl. Math. 43 (1990), 965-981.

11. T. Y. Hou, J. Lowengrub, and Mike Shelley, The exact desingularization of vortex methods and local regridding, Proc. AMS-SIAM Summer Seminar on Vortex Methods and Vortex Dynamics (Seattle, 1990), (C. Anderson and C. Greengard, eds.), SIAM Publication.

12. A. Leonard, Computing three-dimensional incompressible flows with vortex elements, Ann. Rev. Fluid Mech. 17 (1985), 523-559.

13. A. Majda, Vortex dynamics: Numerical analysis, scientific computing, and mathematical theory, Proc. 1st Internat. Congr. in Applied Mathematics, Paris 1987.

14. M. Perlman, On the accuracy of vortex methods, J. Comput. Phys. 59 (1985), 200-223.

15. P. A. Raviart, An analysis of particle methods, CIME Course, Como, Italy, 1983.

16. L. Rosenhead, The point vortex approximation of a vortex sheet, Proc. Roy. Soc. London Ser. A 134 (1932), 170-192.

Courant Institute of Mathematical Sciences, New York University, 251 Mercer STREet, New York, New York 10012

E-mail address: hou@math14.cims.nyu.edu 